\title{
Brilliant Blue G, a P2X7 receptor antagonist, attenuates early phase of renal inflammation, interstitial fibrosis and is associated with renal cell proliferation in ureteral obstruction in rats
}

\author{
José Monteiro Sad Pereira
}

Universidade Federal do Rio de Janeiro Hospital Universitario Clementino Fraga Filho André Luis Barreira

Universidade Federal do Rio de Janeiro Hospital Universitario Clementino Fraga Filho Conrado Rodrigues Gomes

Universidade Federal do Rio de Janeiro Hospital Universitario Clementino Fraga Filho Felipe Mateus Ornellas

Universidade Federal do Rio de Janeiro Hospital Universitario Clementino Fraga Filho Débora Santos Ornellas

Universidade Federal do Rio de Janeiro Hospital Universitario Clementino Fraga Filho

Luiz Carlos Miranda

Universidade Federal do Rio de Janeiro Hospital Universitario Clementino Fraga Filho

\section{Lucio Ronaldo Cardoso}

Universidade Federal do Rio de Janeiro Hospital Universitario Clementino Fraga Filho

\section{Robson Coutinho-Silva}

Universidade Federal do Rio de Janeiro Hospital Universitario Clementino Fraga Filho

\section{Alberto Schanaider}

Universidade Federal do Rio de Janeiro Hospital Universitario Clementino Fraga Filho

\section{Marcelo M. Morales}

Universidade Federal do Rio de Janeiro Hospital Universitario Clementino Fraga Filho

Maurilo Leite ( $\square$ mleitejr@gmail.com )

Universidade Federal do Rio de Janeiro Instituto de Puericultura e Pediatria Martagao Gesteira https://orcid.org/0000-0003-2030-598X

\section{Christina Maeda Takiya}

Universidade Federal do Rio de Janeiro Hospital Universitario Clementino Fraga Filho

\section{Research article}

Keywords: renal inflammation, P2X7 receptor, unilateral ureteral obstruction, macrophages 
Posted Date: November 1st, 2019

DOl: https://doi.org/10.21203/rs.2.16699/v1

License: (c) (i) This work is licensed under a Creative Commons Attribution 4.0 International License. Read Full License

Version of Record: A version of this preprint was published at BMC Nephrology on May 29th, 2020. See the published version at https://doi.org/10.1186/s12882-020-01861-2. 


\section{Abstract}

Background Previous study showed that purinergic P2X7 receptors (P2X7R) reach the highest expression in the first week after unilateral ureteral obstruction (UUO) in mice, and are involved in the process of inflammation, apoptosis and fibrosis of renal tissue. We, herein, document the role of purinergic P2X7 receptors activation on the third day of UUO, as assessed by means of BBG as its selective inhibitor.

Methods We investigated the effects of brilliant blue G (BBG), a P2X7R antagonist, in the third day of kidney tissue response to UUO in rats. For this purpose, male Wistar rats submitted to UUO or sham operated, received BBG or vehicle (V), comprising four groups: UUO-BBG, UUO-V, sham-BBG and sham-V. The kidneys were harvested on day $3 \mathrm{UUO}$ and prepared for histology, immunohistochemistry (P2X7R, PCNA, CD-68, a-sma, TGF- $\beta 1$, Heat-shock protein-47, TUNEL assay), quantitative real-time PCR (IL-1 $\beta$, procollagens type I, III, and IV) for mRNA quantification.

Results The group UUO-V presented an enhancement in tubular cell P2X7-R expression, increase influx of macrophages and myofibroblasts, HSP-47 and TGF- $\beta 1$ expression. Also, upregulation of procollagen types I, III, and IV, and IL-1 $\beta$ mRNAs were seen. On the other hand, group UUO-BBG showed lower expression of procollagens and IL-1 $\beta$ mRNAs, as well as less immunoreactivity of HSP-47, TGF- $\beta$, macrophages, myofibroblasts, and tubular apoptosis. This group also presented increased epithelial cell proliferation.

Conclusion BBG, a known highly selective inhibitor of P2X7R, attenuated renal inflammation, collagen synthesis, renal cell apoptosis, and enhanced renal cell proliferation in the early phase of rat model of UUO.

\section{Background}

Adenosine triphosphate (ATP) is a multifunctional nucleotide, released by injured/dying cells, and is the principal agonist for purinergic $\mathrm{P} 2$ receptors[1]. These receptors are divided into metabotropic $\mathrm{G}$ proteincoupled P2Y (P2YR) and ionotropic ligand gated P2X (P2XR). P2X are ligand-gated ion channels for Na+, $\mathrm{Ca}+$ and $\mathrm{K}+$, known as ionotropic. Currently, seven subtypes of $\mathrm{P} 2 \mathrm{X}$ receptors have been cloned and identified as P2X1-7 [2]. P2X7R are ATP-gated nonselective ion channels, permeable to $\mathrm{Na}+, \mathrm{K}+$, and $\mathrm{Ca} 2+$, expressed in a wide range of epithelial, endothelial, mesenchymal and immune cells. They are ubiquitously expressed in cortex and medulla, in vascular and tubular compartments [3]. P2X7R is scantly expressed in renal tissue in normal conditions, but can be upregulated in disease states [4, 5]. P2XR-ATP axis is important in homeostasis of diverse physiological and pathophysiological processes, including hypertension [6], diabetes [7, 8], polycystic kidney disease [9], inflammatory, and autoimmune disorders [10]. The activation of P2X7Rs may be involved in renal diseases and are widespread in renal compartments, expressed in immune cells, fibroblasts and myofibroblasts, upregulated in inflammation, and associated with the production of pro-inflammatory mediators [11, 12]. 
The progression of chronic kidney disease (CKD) is related to the intensity of renal interstitial fibrosis, the accumulation of extracellular matrix proteins and the process of renal cell death [13]. The importance of P2X7R in renal tissue fibrosis has been highlighted on P2X7R knockout mice submitted to UUO, a wellknown model of tubulointerstitial fibrosis [5].

In the present study, we attempted to investigate the effect of BBG, a selective P2X7R antagonist, on the early development of renal injury after UUO in rats, in order to better elucidate the role of purinergic signaling antagonism on the processes of renal inflammation, fibrosis, renal cells apoptosis and regenerative proliferation in this setting.

\section{Methods}

Forty male adult Wistar rats were housed under specific pathogen-free conditions, with controlled temperature and relative humidity, and provided standard rat chow and water ad libitum. This study was approved by the Animal's Ethics Committee from the Health Sciences Center, Federal University of Rio de Janeiro and is in compliance with the guidelines as recommended by the National Research Council's criteria (NIH No. 86-23).

\section{Experimental Protocol}

Rats were randomly divided into four groups of 5 animals each. Two groups were submitted to a complete UUO and received BBG (UUO-BBG) or vehicle (UUO-V). The other two groups, SHAM-operated, received BBG or vehicle (SHAM-BBG or SHAM-V, respectively).

\section{Surgical Procedure}

Animals were anesthetized with ketamine $(35 \mathrm{mg} / \mathrm{kg})$ and xylazine $(9 \mathrm{mg} / \mathrm{kg})$ by peritoneal route. An abdominal midline incision was done and the left ureter was ligated at two points using 4-0 silk and sectioned. BBG (Brilliant Blue G), 40 mg/kg (Sigma-Aldrich, Saint Louis, MO, USA, cat. B0770) was dissolved in $0.2 \%$ dimethyl sulfoxide (DMSO, Sigma-Aldrich, cat. D2650) in sterile saline solution and was injected in the inferior cava vein $(0.5 \mathrm{~mL})$ after ureteral ligation (Group UUO-BBG). In another group, obstructed rats received vehicle instead of BBG (Group UUO-V). The abdominal wall was closed layers and the rats were kept in regular cages. The sham-operated animals underwent identical surgical procedures, but without ligation and sectioning of the left ureter.

\section{Tissue preparation}

After 3 days following the surgical procedure, animals were sacrificed under anesthesia by means of cardiac puncture and perfusion in a controlled flow rate of $10 \mathrm{~mL} / \mathrm{min}$ of a sterile $0.9 \%$ saline solution 
with heparin $(5 \mathrm{U} / \mathrm{mL})$, infused through the left ventricle to clean the blood vessels. The left kidney was stored for further analysis.

\section{Histopathological and Immunohistochemical studies}

The kidneys were embedded in paraffin after fixation, sectioned in 3- $\mu \mathrm{m}$ width slices and stained for picro-sirius red (PS) assuming collagen quantification. Immunohistochemical procedures were performed on paraffın-embedded kidney sections. After dewaxing and rehydrating, sections were submitted to endogenous peroxidase inhibition. Heat mediated-antigen retrieval and enzymatic techniques were performed according to the specific antibody. After blocking the nonspecific binding of immunoglobulins to the tissue, primary antibodies were incubated overnight at $4^{\circ} \mathrm{C}$ in a humidified chamber for about $16 \mathrm{~h}$. The secondary antibodies were incubated (Histofine ${ }^{\circledR}$ Simple Stain Rat MAX - PO (Mouse), and - PO (Rabbit) from Nichirei, Japan). The chromogen substrate was diaminobenzidine (Liquid DAB, Dako, cat. K3468). For P2X7R staining, P2X7R antibody incubation was performed using the P2X7R peptide (control antigen for APR-004, Alomone) for $1 \mathrm{~h}$ at room temperature. This antibody solution was incubated on sections instead of the P2X7R antibody alone.

\section{Histomorphometry}

Images were captured by a light microscope (Eclipse E800, Nikon, Japan) coupled to a digital camera and analyzed using the Image Pro Plus software (version 4.5.1, Media Cybernetics, Rockville, MD, USA).

\section{Picro Sirius red, P2X7R, macrophage (CD68), myofibroblast (a-SMA), Heat Shock Protein 47 (HSP-47), and TGF- $\beta 1$ surface density quantifications}

Tissue sections were used to obtain 20 randomly chosen photomicrographs from renal cortex and medulla, avoiding fields containing blood vessels and glomeruli. Objective lens with a magnification of 40x was used. Results were expressed as surface densities in the cortex or medulla and were expressed as percentages mean \pm standard error of the mean (SEM).

\section{Collagen content, and IL-1 $\beta$ in renal tissue by quantitative real-time reverse transcription-polymerase chain reaction (qRT-PCR)}

Total ribonucleic acid (RNA) was extracted from snap frozen renal tissues using the spin total RNA Isolation System (Promega Corporation, Fitchburg, WI, USA) following manufacturer's recommendations. RNA concentration was measured by spectrophotometry in Nanodrop ND-1000. First-strand cDNA was synthesized from total RNA using GoTaq 2-STEP RT qPCR System (Promega Corporation). The primers sequences for collagen content, and IL-1 $\beta$ are depicted in table 1. Relative messenger RNA (mRNA) 
levels were measured with a SYBR green detection system using Mastercycler RealPlex 2 (Eppendorf, Hamburg, Germany). Samples were measured in triplicate. Ciclophilin was used as internal control.

\section{Renal cells apoptosis}

Apoptotic tubular cells in kidney tissue were detected by the terminal deoxytransferase uridine triphosphate nick end-labeling technique (TUNEL), using ApopTag® Peroxidase in situ detection kit (Chemicon International, Temecula, CA, USA). The reaction was performed according to the manufacturer's instructions, revealed with diaminobenzidine (Liquid DAB, Dako). Results were expressed as the percentage of positive tubular cells in a total of 100 cells.

\section{Proliferation index}

Proliferation index was obtained by the ratio of tubular cells positive for PCNA (Proliferating cell nuclear antigen), divided by the total number of tubular cells. Results were expressed as the percentage of positive tubular cells.

\section{Statistical analysis}

The results obtained from 20 randomly chosen photomicrographs of each animal, in each immunohistochemical study, presented as normal distribution. Results are expressed as mean \pm SEM. Statistical analysis was performed using ANOVA followed by the Student Neuman Keuls test. $P<0.05$ was considered statistically significant.

\section{Results}

\section{Histopathological study}

Hematoxylin-eosin-stained sections of UUO animals showed interstitial enlargement and dilated tubular structures. There was no statistical difference of tubular and interstitial areas of the renal tissue between UUO-V and UUO-BBG groups. Sham animals did not show significant histological alterations (data not shown).

\section{P2X7R expression}

Histomorphometrical analysis of P2X7R showed increased immunostaining in UUO groups compared to Sham groups in renal cortex $(0.041 \pm 0.004$ vs $0.007 \pm 0.004$; UUO-V vs Sham-V, respectively; $p<0.05)$ and $(0.03 \pm 0.001$ vs $0.008 \pm 0.006$, UUO-BBG vs Sham-BBG, respectively; $p<0.05)$. BBG decreased the surface density of the P2X7R in UUO in renal cortex $(0.041 \pm 0.01$ vs $0.030 \pm 0.001$; UUO-V vs UUO-BBG, respectively, $\mathrm{p}<0.05)$ (Figure $1 \mathrm{~A})$, but not in renal medulla $(0,031 \pm 0,004$ vs $0,025 \pm 0,003$; OUU-V vs OUU-BBG, 
respectively, $p>0.05$ ) (figure 1D). UUO-V groups showed more immunoreactive tubules on renal cortex, but not on medulla, compared to UUO-BBG groups (Figure 1).

\section{BBG attenuates macrophage infiltration and downregulates the expression of IL-1 $\beta$ mRNA}

Macrophages were immunostained with the anti-CD-68 antibody, and located in interstitial spaces, around glomerular capsule and blood vessels. UUO-BBG animals showed a decrease of about $85 \%$ of anti-CD -68 antibody surface density compared to UUO-V animals in renal cortex $(0.013 \pm 0.002 \mathrm{vs}$ $0,003 \pm 0$; UUO-V vs UUO-BBG, respectively; $p<0.0001$; Figure $2 A, 2 B, 2 C)$ and medulla $(0.015 \pm 0.003$ vs $0.002 \pm 0.001$; UUO-V vs UUO-BBG, respectively; $p<0.0001$; Figures $2 D, 2 E, 2 F)$. Tissue samples from UUO animals treated with BBG showed a reduction of about $60 \%$ of $\mathrm{IL}-1 \beta$ mRNA expression compared to UUO-V animals ( $2.9 \pm 0.8$ vs $0.4 \pm 0.08$; UUO-V vs OUU-BBG, respectively; $P<0.0001$; figure $2 \mathrm{G}$ ).

\section{BBG attenuates myofibroblast infiltration and Heat Shock protein 47 expression}

The surface density of a-smooth muscle actin in tissue samples from UUO-BBG animals showed about $80 \%$ reduction of myofibroblast surface density in renal cortex $(0.045 \pm 0.004$ vs $0.007 \pm 0.002 ;$ UUO-V vs UUO-BBG, respectively; $p<0.0001)$ and medulla $(0.060 \pm 0.3$ vs $0.005 \pm 0.001 ;$ UUO-V vs UUO-BBG, respectively; $p<0.0001$ ), compared to UUO-V animals (Figures 3A-F). HSP47 immunostaining was performed to analyze collagen synthesis. HSP47 immunostained area was reduced about $85 \%$ in UUOBBG group compared to UUO-V animals in renal cortex $(0.029 \pm 0.008$ vs $0.004 \pm 0.001$; UUO-V vs OUU-BBG, respectively; $P<0.0001)$ (Figures $3 G-1)$ and medulla ( $0.029 \pm 0.7$ vs $0.004 \pm 0.001$; UUO-V vs OUU-BBG, respectively; $\mathrm{P}<0.0001$ ) (Figures $3 \mathrm{~J}-\mathrm{L}$ ).

\section{BBG attenuates collagen deposition and gene expression of procollagen I, III, and IV}

The effect of P2X7R blockage on the early stage of UUO-induced collagen deposition in renal tissue was assessed by picro-sirius red staining. UUO-V animals showed the highest level of collagen compared to all other groups in renal cortex and medulla. UUO-BBG rats presented nearly $60 \%$ less collagen compared to UUO-V group, in renal cortex ( $0.067 \pm 0.004$ vs $0.025 \pm 0.003$; UUO-V vs UUO-BBG, respectively; $p<0.05$ ) (Figures 4A-C). The same pattern was observed in renal medulla $(0.062 \pm 0.004$ vs $0.025 \pm 0.003$; UUO-V vs UUO-BBG, respectively; $p<0.05$ ) (Figures 4D-F).

The qRT-PCR technique was performed to evaluate the expression of procollagen I, III and IV mRNA at day 3 of UUO. When comparing groups UUO-BBG and UUO-V, there was reduction of the expression of procollagen I mRNA (20.2 \pm 2.4 vs $13.4 \pm 2$; UUO-V vs UUO-BBG, respectively; $p=0.0001)$ (Figure $4 G$ ), and 
procollagen III mRNA (13.62 \pm 1.1 vs $8.4 \pm 0.75$; UUO-V vs UUO-BBG, respectively; $p<0.0001)$ (Figure $4 \mathrm{H}$ ). Moreover, procollagen IV mRNA was reduced in about 70\% in UUO-BBG compared to UUO-V group $(12.4 \pm 2.2$ vs $3.4 \pm 0.5$; UUO-V vs UUO-BBG, respectively; $p<0.01$ ) (Figure $4 I$ ).

\section{BBG downregulates the immunoexpression of TGF- $\beta$}

TGF- $\beta$ was present in cortical and medullar renal tubules as well as in mononuclear inflammatory cells and vessels walls. Only the tubular TGF- $\beta$ immunostaining was considered in the histomorphometric analysis. Renal tubules from UUO-BBG group showed a 75\% reduction of TGF- $\beta$ immunoexpression compared to UUO-V group in renal cortex $(0.04 \pm 0.01$ vs $0.01 \pm 0.010$; OUU-V vs OUU-BBG, respectively; $p=$ 0.0008 ) (Figures $5 \mathrm{~A}-\mathrm{C})$ and a reduction of about $60 \%$ in renal medulla $(0.06 \pm 0.01$ vs $0.02 \pm 0.01$; OUU-V vs OUU-BBG, respectively; $p<0.05$ ) (Figures 5D-F).

\section{BBG increases tubular cell regeneration and attenuates apoptosis}

The effect of BBG on proliferation and loss of tubular cells by apoptotic cell death at day 3 of UUO were evaluated by immunohistochemistry for the PCNA antigen and the TUNEL assay, respectively. UUO-BBG group showed significant higher tubular cell regeneration compared to UUO-V group in the cortex (about $35 \%)(18.4 \pm 3.4$ vs $25.0 \pm 1.7$; UUO-V vs UUO-BBG, respectively; $\mathrm{p}<0.0001$ ) and medulla (about $80 \%$ ) (15.9 \pm 2.68 vs $28.8 \pm 2.6$; UUO-V vs UUO-BBG, respectively; $p<0.05$ ) (Figures $6 \mathrm{~A}-\mathrm{F}$ ). Furthermore, UUO-BBG group showed about $70 \%$ less tubular cell apoptosis compared to UUO-V group in the cortex $(48.5 \pm 3.08$ vs $16 \pm 4.78$; UUO-V vs UUO-BBG, respectively; $p<0.0001)$, and medulla $(51,8 \pm 6,25$ vs $14,5 \pm 5,09$ OUU-V vs OUU-BBG, respectively; $p<0.05$ ), (Figures 6G-L).

\section{Discussion}

Several lines of evidence suggest that purinergic P2X7 receptors participate in the processes of various kidney diseases $[14,15,16,17]$. In several previous studies, BBG, a selective P2X7R antagonist, was used as a potent inhibitor of P2X7R that reduces inflammation, immune cells activation, and fibrosis $[10,18$, 19]. BBG was able to reduce renal injury in Dahl salt-sensitive hypertensive rats and in lupus nephritis model $[6,10]$ similarly to P2X7R null mice [5]. Of note, BBG also antagonizes rat P2X4Rs, but its selectivity for P2X7R is 1000 -fold greater $[20,21]$. The present study addressed the effects of BBG on the initial process of renal interstitial inflammation, collagen deposition, renal cell apoptosis and proliferation, by using the model of unilateral ureteral obstruction. Our results showed that BBG attenuated renal damage, similar to those in P2X7R knockout mice [5] and in other disease models $[10,14,18,19,22,23]$.

Our previous study about the effects of $\mathrm{P} 2 \mathrm{X} 7$ receptors used the same fibrogenic model of renal disease [5]. In that study, we used knockout mice for P2X7R and we observed that these receptors could not be seen as constitutively expressed, but only in obstructed groups, probably localized on epithelial tubular cells. In addition, P2X7R were apparent only on day 7 of UUO and not on day 14 . The present study was 
designed to observe the pathophysiological aspect of rat kidneys on day 3 of UUO, in which P2X7R was assumed to be expressed. In fact, P2X7R expression could be clearly seen by the immunohistochemical study, on the aspect of tubular epithelial cells, as well as in some interstitial cells. On the other hand, P2X7R were also expressed on sham-operated animals, although with significantly lower intensity. It is noteworthy that their expression was seen in BBG treated UUO group as significantly lower when compared with non-treated UUO group, an observation also mentioned by Marques et al [18] in another study using BBG. The explanation for the decreased expression of P2X7R on BBG treated group is not apparent at this moment, but one can argue about the possibility of receptor downregulation by BBG antagonism or allosteric modification of the epitope. Future studies are needed to elucidate this issue.

The results on the monocytes/macrophage recruitment after UUO clearly showed the expected increase of renal interstitial inflammation, which was decreased in BBG treated animals (Fig. 2). Nonetheless, perhaps the most specific feature associated with P2X7R activation and the development of tissue inflammation might be the secretion of inflammasome-related cytokines [24, 25]. In this regard, it has been consistently documented that the effect of P2X7R activation is closely linked to IL-1 $\beta$ secretion [24, 26]. In renal tissue, Deplano et al [16] and Jalilian et al [27] have previously demonstrated the role of ATP, as a damage-associated molecular pattern, on the activation of P2X7R to trigger the secretion of IL $-1 \beta$. Our results showed that the increased IL $-1 \beta$ mRNA in UUO tissue kidneys was abrogated in BBG-treated animals (Fig. 2G).

A significant reduction in myofibroblast population (Fig. 3A-F) was observed in BBG treated group. Moreover, an effect on myofibroblast function was strongly suggested from the results of HSP47 (Fig. 3G-L). This chaperone is an endoplasmic reticulum (ER)-resident, stress inducible glycoprotein, collagenspecific heat-shock protein, which plays a key role in collagen biosynthesis and its structural assembly [28]. It is also used as a biomarker to identify collagen-producing cells [29]. A previous study using the model of UUO in mice clearly showed that HSP-47 was overexpressed in the renal interstitium of obstructed animals [30]. In our study, BBG treated group showed decreased expression of the chaperone. Therefore, it is conceivable to suggest that the reduction of HSP -47 stained cells probably indicates a reduction of myofibroblasts function by the purinergic blockage.

The aspect of interstitial collagen deposition is a known striking histopathological feature of the UUO model. In this study, the expression of TGF- $\beta$, the fibrogenic cytokine associated with collagen deposition, was shown to be decreased in BBG treated rats (Fig. 5). Also, decreased collagen deposition, as expressed by picrosirius red staining, was seen in BBG treated group (Fig. 4A-F). Likewise, pro-collagen I, III and IV mRNA were shown to be increased in obstructed animals, with significant reduction in BBG treated rats (Fig. 4G-I).

Our previous study on P2X7 knockout mice clearly evidenced the implication of these receptors on the process of apoptosis of renal cells [5]. In fact, the present study showed the decrease of apoptotic cells in kidneys from BBG treated group. Likewise, renal cells proliferation also increased in the group with BBG, and these results suggest that regenerative process after kidney damage by UUO can be up-regulated by 
P2X7R antagonism (Fig. 6). We have previously demonstrated the action of bone marrow-derived cells to induce and attenuate renal cell proliferation and apoptosis, respectively [31]. Nevertheless, little is known about the involvement of P2X7 receptors activation in this setting. It is noteworthy, however, that Chen et al [32] have found an increase in P2X7R expression after derangement of retinal ganglion cells, which decreased in animals treated with human umbilical cord blood mesenchimal stem cells. This observation suggests a mechanistic antagonism between P2X7R expression and activation, and the action of progenitor cells to determine proliferative repair. In the present study, the putative BBG action to inhibit P2X7R activation, which was related to increased proliferation of renal cells, might suggest a modulatory role of this receptor on the mechanism of renal cell repair after epithelial cell injury.

In summary, the results from this study highlight the beneficial role of P2X7R antagonism, as can be accomplished by BBG, in the prevention of the early phase of inflammation and the ensuing fibrogenic process, even in the third day of UUO. As the previous studies using P2X7R antagonists in various disease models, one can suggest that targeting this receptor might be beneficial in selective conditions. In addition, this study also constitutes evidence that the blockage of purinergic P2X7 receptor may act in favor of renal cell proliferation and tissue regeneration, and the mechanism underlying this effect needs extensive investigation.

\section{Abbreviations}

BBG: brilliant blue G; UUO: unilateral ureteral obstruction; PCNA: Proliferating Cell Nuclear Antigen; TUNEL: Terminal deoxynucleotidyl transferase dUTP nick end labeling; qRT-PCR: reverse transcription polymerase chain reaction quantitative real time; IL-1 $1 \beta$ : interleukin 1 beta: mRNA: messenger ribonucleic acid; ATP: adenosine triphosphate; CKD: chronic kidney disease; DMSO: dimethyl sulfoxide

\section{Declarations}

\section{Ethics approval and consent to participate}

This study was approved by the Animal's Ethics Committee from the Health Sciences Center, Federal University of Rio de Janeiro and is in compliance with the guidelines as recommended by the National Research Council's criteria (NIH No. 86-23). The animals were acquired from the University vivarium and, therefore, an owner consent was not required.

\section{Consent for publication}

Not applicable

\section{Availability of data and material}


The datasets analysed during the current study are available from the corresponding author on reasonable request (mleitejr@gmail.com).

\section{Competing interests}

\section{The authors report no competing interest}

\section{Funding}

This work was supported by funds from Fundação de Amparo à Pesquisa do Estado do Rio de Janeiro (FAPERJ E-26/110.241/2014). The funding was applied specifically to purchase all the reagents used.

\section{Authors contribution}

The contribution of each authors is as follows: (1) JMSP, ALB and CRG conducted the animal surgical procedures and histhopathological studies. (2) FMO and DSO performed qRT-PCR studies. (3) LCM, RCS, AS, MMM and CMT conducted the study conception and design. (4) LRC conducted the statistical analysis and data interpretation. All authors have read and approved the final version of the manuscript.

\section{Aknowledgments}

Not applicable.

\section{References}

1. Vénéreau E, Ceriotti C, Bianchi ME. DAMPs from Cell Death to New Life. Front Immunol. 2015;6:422.

2. Burnstock G. Purinergic signalling. Br J Pharmacol. 2006;147:S172-181.

3. Turner CM, Vonend O, Chan C, Burnstock G, Unwin RJ. The pattern of distribution of selected ATPsensitive P2 receptor subtypes in normal rat kidney: an immunohistological study. Cells Tissues Org. 2003;175:105-117.

4. Hillman KA, Burnstock G, Unwin RJ. The P2X7 ATP receptor in the kidney: a matter of life or death? Nephron Exp Nephrol. 2005;101(1):e24-30.

5. Gonçalves RG, Gabrich L, Rosário A Jr, Takiya CM, Ferreira ML, Chiarini LB, et al. The role of purinergic $\mathrm{P} 2 \mathrm{X7}$ receptors in the inflammation and fibrosis of unilateral ureteral obstruction in mice. Kidney Int. 2006;70:1599-1606.

6. Ji X, Naito Y, Hirokawa G, Weng H, Hiura Y, Takahashi R, et al. P2X(7) receptor antagonism attenuates the hypertension and renal injury in Dahl salt sensitive rats. Hypertens Res. 2012; 35:173-179. 
7. Vonend O, Turner CM, Chan CM, Loesch A, Dell'Anna GC, Srai KS, et al. Glomerular expression of the ATP sensitive P2X7 receptor in diabetic and hypertensive rat models. Kidney Int. 2004;66:157-166.

8. Solini A1, Chiozzi P, Morelli A, Adinolfi E, Rizzo R, Baricordi OR, et al. Enhanced P2X7 activity in human fibroblasts from diabetic patients: a possible pathogenetic mechanism for vascular damage in diabetes. Arterioscler Thromb Vasc Biol. 2004;24(7):1240-1245.

9. Turner CM, Ramesh B, Srai SK, Burnstock G, Unwin RJ. Altered ATPsensitive P2 receptor subtype expression in the Han:SPRD cy/+ rat, a model of autosomal dominant polycystic kidney disease. Cells Tissues Organs. 2004;178:168-179.

10. Zhao J, Wang $\mathrm{H}$, Dai C, Wang $\mathrm{H}$, Zhang $\mathrm{H}$, Huang $\mathrm{Y}$, et al. P2X7 Blockade Attenuates Murine Lupus Nephritis by Inhibiting Activation of the NLRP3/ASC/Caspase 1 Pathway. Arthritis Rheum. 2013;65:3176-3185.

11. Lu D, Insel PA. Cellular mechanisms of tissue fibrosis. 6. Purinergic signaling and response in fibroblasts and tissue fibrosis. Am J Physiol Cell Physiol. 2014;306:C779-788.

12. Ferrari D, Pizzirani C, Adinolfi E. The P2X7 receptor: a key player in IL-1 processing and release. J Immunol. 2006;176:3877-3883.

13. Eddy AA, Neilson EG. Chronic kidney disease progression. J Am Soc Nephrol. 2006;17:2964-2966.

14. Solini A, Menini S, Rossi C, Ricci C, Santini E, Blasetti Fantauzzi C, et al. The purinergic 2X7 receptor participates in renal inflammation and injury induced by high-fat diet: possible role of NLRP3 inflammasome activation. J Pathol. 2013;231:342-353.

15. Yan Y, Bai J, Zhou X, Tang J, Jiang C, Tolbert E, et al. P2X7 receptor inhibition protects against ischemic acute kidney injury in mice. Am J Physiol Cell Physiol. 2015;15:308(6):C463-472.

16. Deplano S, Cook HT, Russell R, Franchi L, Schneiter S, Bhangal G, et al. P2X7 receptor-mediated Nlrp3-inflammasome activation is a genetic determinant of macrophage-dependent crescentic glomerulonephritis. J Leukoc Biol. 2013;93(1):127-134.

17. Taylor SR, Turner CM, Elliott JI, McDaid J, Hewitt R, Smith J, et al. P2X7 deficiency attenuates renal injury in experimental glomerulonephritis. J Am Soc Nephrol. 2009;20(6):1275-1281.

18. Marques CC, Castelo-Branco MT, Pacheco RG, Buongusto F, do Rosário A Jr, Schanaider A, et al. Prophylactic systemic P2X7 receptor blockade prevents experimental colitis. Biochim Biophys Acta. 2014;1842:65-78.

19. Peng W, Cotrina ML, Han X, Yu H, Bekar L, Blum L, et al. Systemic administration of an antagonist of the ATP-sensitive receptor P2X7 improves recovery after spinal cord injury. Proc Natl Acad Sci USA. 2009;106:12489-12493.

20. Menzies RI, Unwin RJ, Dash RK, Beard DA, Cowley AW Jr, Carlson BE, et al. Effect of P2X4 and P2X7 receptor antagonism on the pressure diuresis relationship in rats. Front Physiol. 2013;4: 305.

21. Jiang LH, Mackenzie AB, North RA, Surprenant A. Brilliant blue G selectively blocks ATP-gated rat P2X(7) receptors. Mol Pharm. 2000;58:82-88. 
22. Monção-Ribeiro LC, Faffe DS, Santana PT, Vieira FS, da Graça CL, Marques-da-Silva C, et al. P2X7 receptor modulates inflammatory and functional pulmonary changes induced by silica. PLoS One. 2014;9:e110185.

23. Martins JP, Silva RB, Coutinho-Silva R, Takiya CM, Battastini AM, Morrone FB, et al. The role of P2X7 purinergic receptors in inflammatory and nociceptive changes accompanying cyclophosphamideinduced haemorrhagic cystitis in mice. Br J Pharmacol. 2012; 165:183-196.

24. Qu Y, Franchi L, Nunez G, Dubyak GR. Nonclassical IL-1 beta secretion stimulated by P2X7 receptors is dependent on inflammasome activation and correlated with exosome release in murine macrophages. J Immunol. 2007;179(3):1913-1925.

25. Bours MJ, Dagnelie PC, Giuliani AL, Wesselius A, Di Virgilio F. P2 receptors and extracellular ATP: a novel homeostatic pathway in inflammation. Front Biosci (Schol Ed). 2011;3:1443-1456.

26. Lee BH, Hwang DM, Palaniyar N, Grinstein S, Philpott DJ, Hu J. Activation of P2X(7) receptor by ATP plays an important role in regulating inflammatory responses during acute viral infection. PLoS One. 2012;7(4):e35812.

27. Jalilian I, Spildrejorde M, Seavers A, Curtis BL, McArthur JD, Sluyter R. Functional expression of the damage-associated molecular pattern receptor P2X7 on canine kidney epithelial cells. Vet Immunol Immunopathol. 2012; 150(3-4):228-233.

28. Mala JG, Rose C. Interactions of heat shock protein 47 with collagen and the stress response: an unconventional chaperone model? Life Sci. 2010;87:579-586.

29. Taguchi T, Nazneen A, Al-Shihri AA, Turkistani KA, Razzaque MS. Heat shock protein 47: a novel biomarker of phenotypically altered collagen-producing cells. Acta Histochem Cytochem. 2011;44:3541.

30. Moriyama T, Kawada N, Ando A, Yamauchi A, Horio M, Nagata K, et al. Up-regulation of HSP47 in the mouse kidneys with unilateral ureteral obstruction. Kidney Int. 1998;54(1):110-119.

31. Barreira AL, Takiya CM, Castiglione RC, Maron-Gutierrez T, Barbosa CM, Ornellas DS, et al. Bone marrow mononuclear cells attenuate interstitial fibrosis and stimulate the repair of tubular epithelial cells after unilateral ureteral obstruction. Cell Physiol Biochem. 2009;24(5-6):585-594.

32. Chen M1, Xiang Z, Cai J. The anti-apoptotic and neuro-protective effects of human umbilical cord blood mesenchymal stem cells (hUCB-MSCs) on acute optic nerve injury is transient. Brain Res. 2013;1532:63-75.

\section{Table 1}

Table 1 Base sequences of primers for Procollagens I, II and IV, IL-1 $\beta$ and ciclophilin 


\begin{tabular}{lll}
\hline \multicolumn{1}{c}{ Genes } & \multicolumn{1}{c}{ Sense } & \multicolumn{1}{c}{ Anti-sense } \\
\hline Procollagen I & 5'TGGAATCTTGGATGGTTTGGA 3' & 5' GCTGTAAACGTGGAAGCAAGG 3' \\
Procollagen III & 5' ACCTGGACCACAAGGACAC 3' & 5' TGGACCCATTTCACCTTTC 3' \\
Procollagen IV & 5' ATTCCTTTGTGATGCACACCAG 3' & 5' AAGCTGTAAGCATTCGCGTAGA 3' \\
IL-1 $\beta$ & 5'-CTATGTCTTGCCCGTGGAG-3 & 5'-CATCATCCCACGAGTCACA-3' \\
Ciclophilin & 5'-TCCACTTCGATCTTGCCACAGTCT-3' & 5'-AGACACCAATGGCTCCCAGTTCTT-3' \\
\hline
\end{tabular}

\section{Figures}

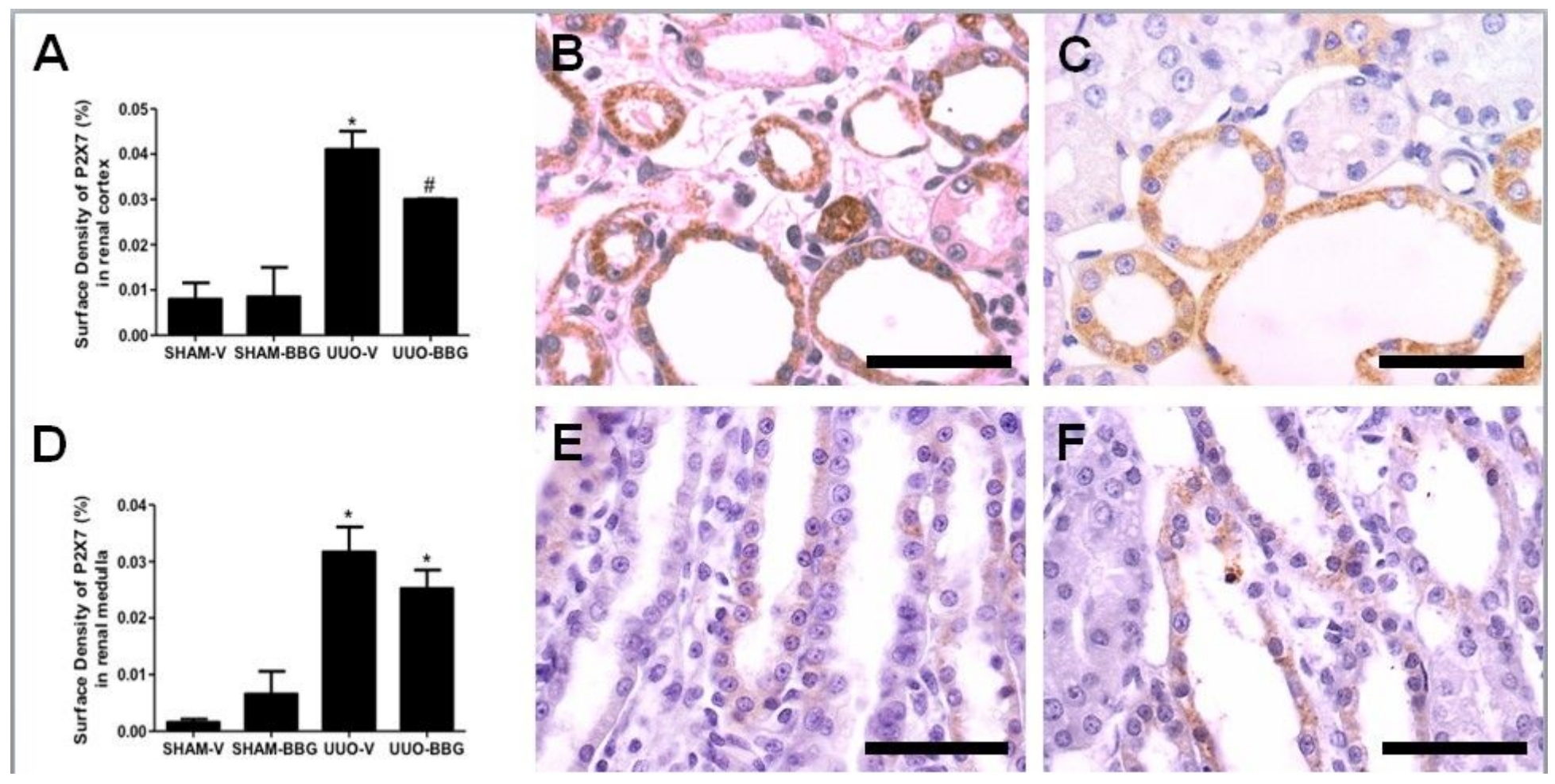

Figure 1

BBG administration decreases P2X7R immunoexpression in tubular cortex but not in medulla in UUO. a Graphical representation of P2X7R surface density in tubular cells in renal cortex. Columns indicate means \pm SEM of 20 captured images of each kidney in the different experimental groups. ${ }^{*}<0.05$ vs UUO-BBG and both SHAM groups. \# $\mathrm{p}<0.05$ vs UUO-V and both SHAM groups. b Representative immunostaining of the renal cortex from UUO-V group. c UUO-BBG group. $d$ Graphical representation of P2X7R surface density in tubular cells in renal medulla. Columns indicate means \pm SEM of 20 captured images of each kidney in the different experimental groups. * $p<0.05$ vs both SHAM groups, but not between UUO groups. e Representative immunostaining of the renal medulla from UUO-V group and $f$ UUO-BBG group $(B a r=50 \mu \mathrm{m})$ 
A

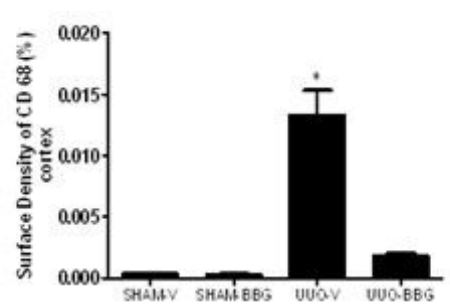

G
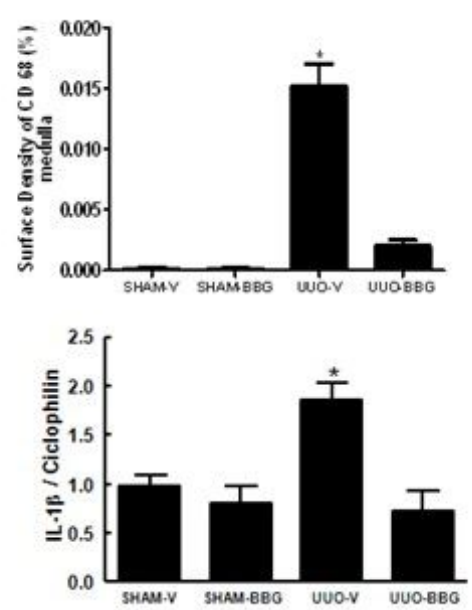
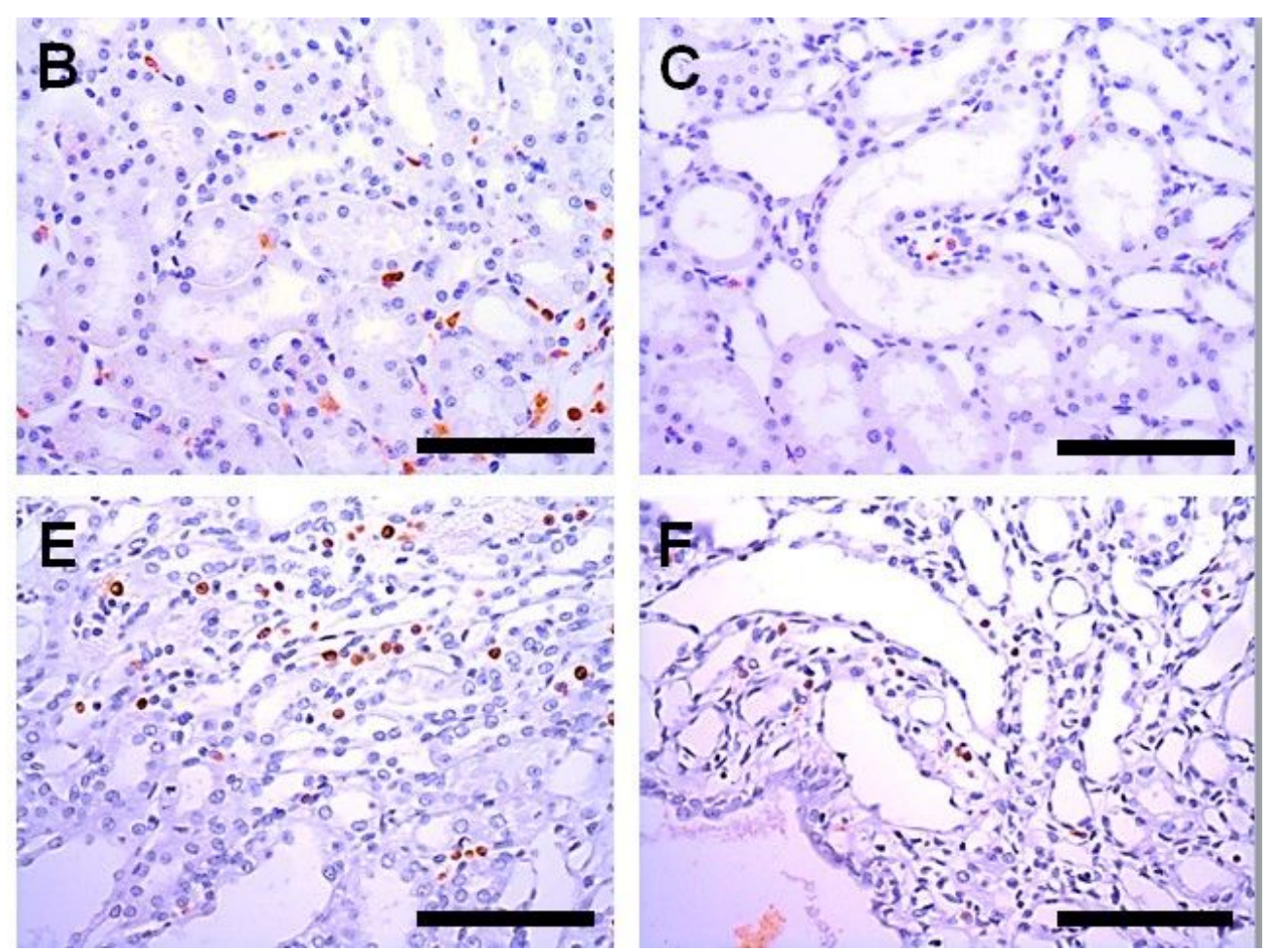

Figure 2

BBG and inflammation. BBG reduces the infiltration of macrophages in the renal interstitium and the mRNA expression of IL1- $\beta$ in UUO. a Graphical representation of CD68 surface density in tubular cells in renal cortex. Columns indicate means \pm SEM of 20 captured images of each kidney in the different experimental groups. * $p<0.05$ vs UUO-BBG and both SHAM groups. b Representative immunostaining of the renal cortex from UUO-V group. c UUO-BBG group. $d$ Graphical representation of CD68 surface density in tubular cells in renal medulla. Columns indicate means \pm SEM of 20 captured images of each kidney in the different experimental groups. * $p<0.05$ vs UUO-BBG and both SHAM groups. e Representative immunostaining of the renal medulla from UUO-V group and f UUO-BBG group. (Bar $=100 \mu \mathrm{m}) . \mathrm{g}$ Graphical representation of the mRNA expression of IL1- $\beta$ by semiquantitative RT-PCR. Quantification of densitometric values obtained from the ratio IL1- $\beta$ /Ciclophilin (mean $\pm S E M, n=6$ ) under the 4 experimental conditions indicated on the abscissa. ${ }^{*} p<0.05$ vs UUO-BBG and both SHAM groups 
A

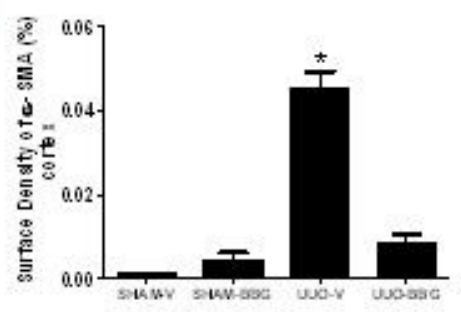

D

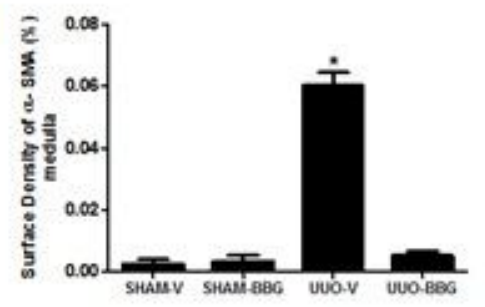

G

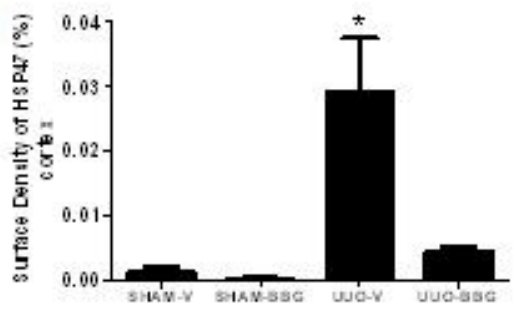

$J$

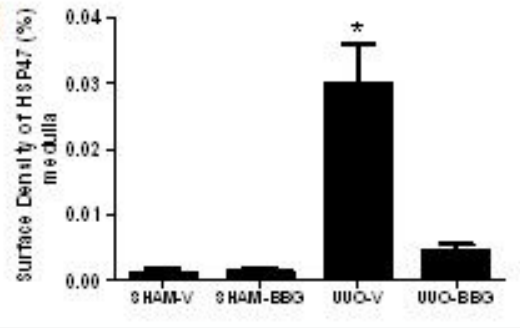

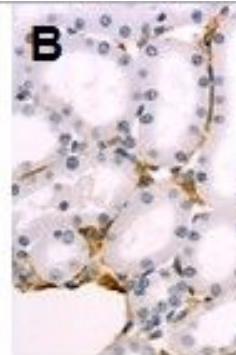
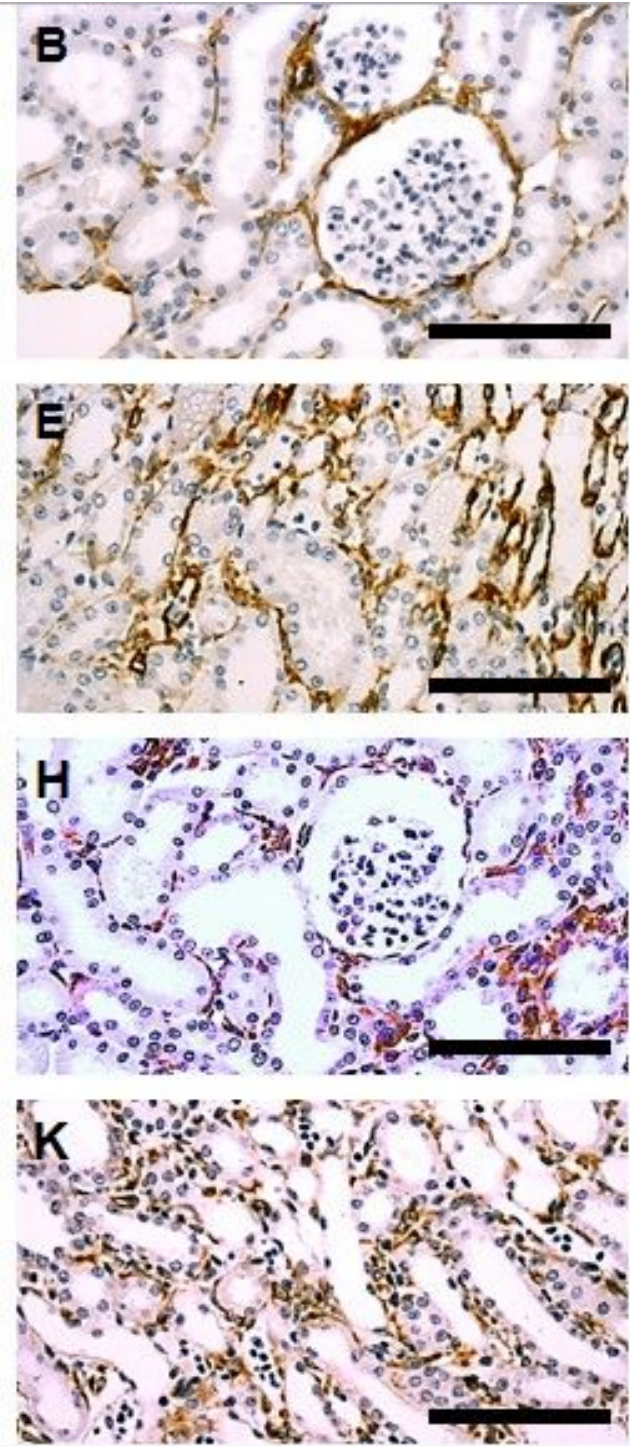
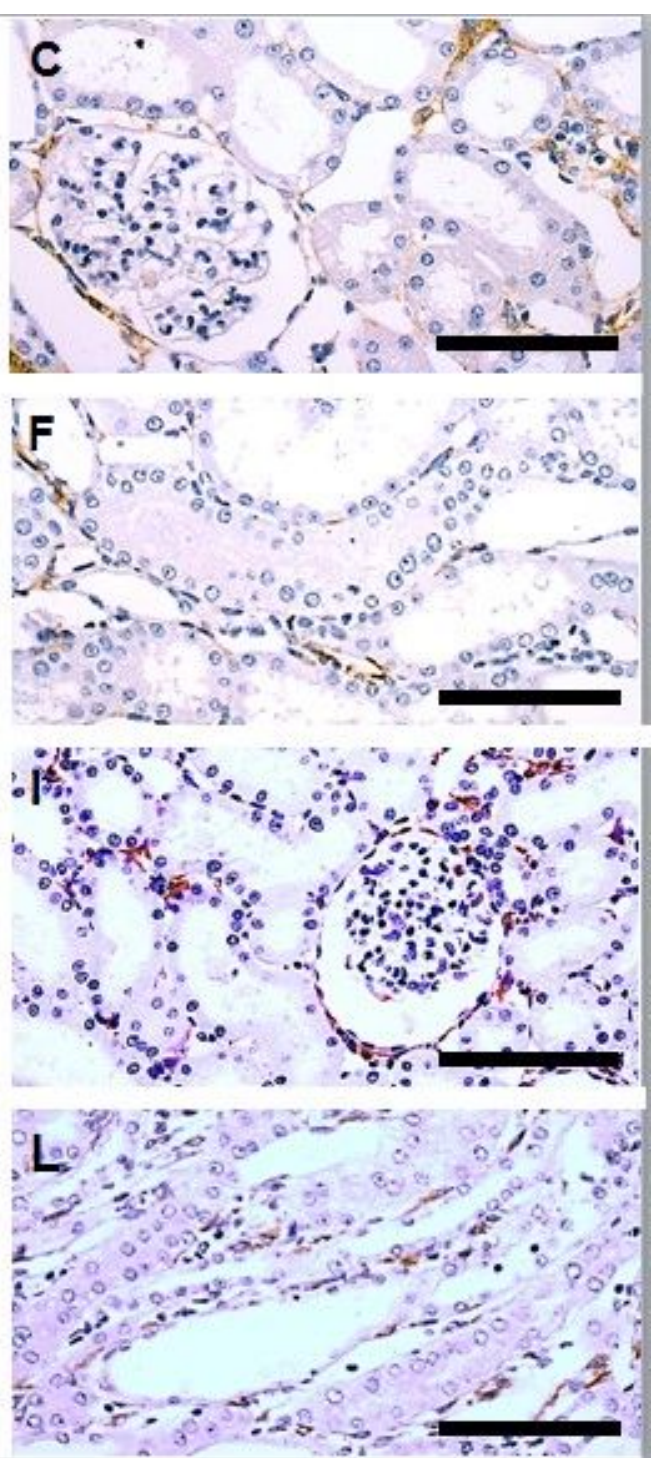

Figure 3

BBG reduces the pool of myofibroblasts and collagen synthesis in UUO. a Graphical representation of aSMA surface density in renal cortex. Columns indicate means \pm SEM of 20 captured images of each kidney in the different experimental groups. * $p<0.05$ vs UUO-BBG and both SHAM groups. $b$ Representative immunostaining of the renal cortex from UUO-V group and c UUO-BBG group. d Graphical representation of a-SMA surface density in renal medulla. Columns indicate means \pm SEM of 20 captured images of each kidney in the different experimental groups. * $p<0.05$ vs UUO-BBG and both SHAM groups. e Representative immunostaining of the renal medulla from UUO-V group and $f$ UUO-BBG group. $\mathrm{g}$ Graphical representation of HSP-47 surface density in renal cortex. Columns indicate means \pm SEM of 20 captured images of each kidney in the different experimental groups. * $p<0.05$ vs UUO-BBG and both SHAM groups. h Representative immunostaining of the renal cortex from UUO-V group and i UUO-BBG group. j Graphical representation of HSP-47 surface density in renal medulla. Columns indicate means \pm SEM of 20 captured images of each kidney in the different experimental groups. * $p<0.05$ vs UUO-BBG and both SHAM groups. $\mathrm{k}$ Representative immunostaining of the renal medulla from UUO-V group and I UUO-BBG group. $($ Bar $=100 \mu \mathrm{m})$ 


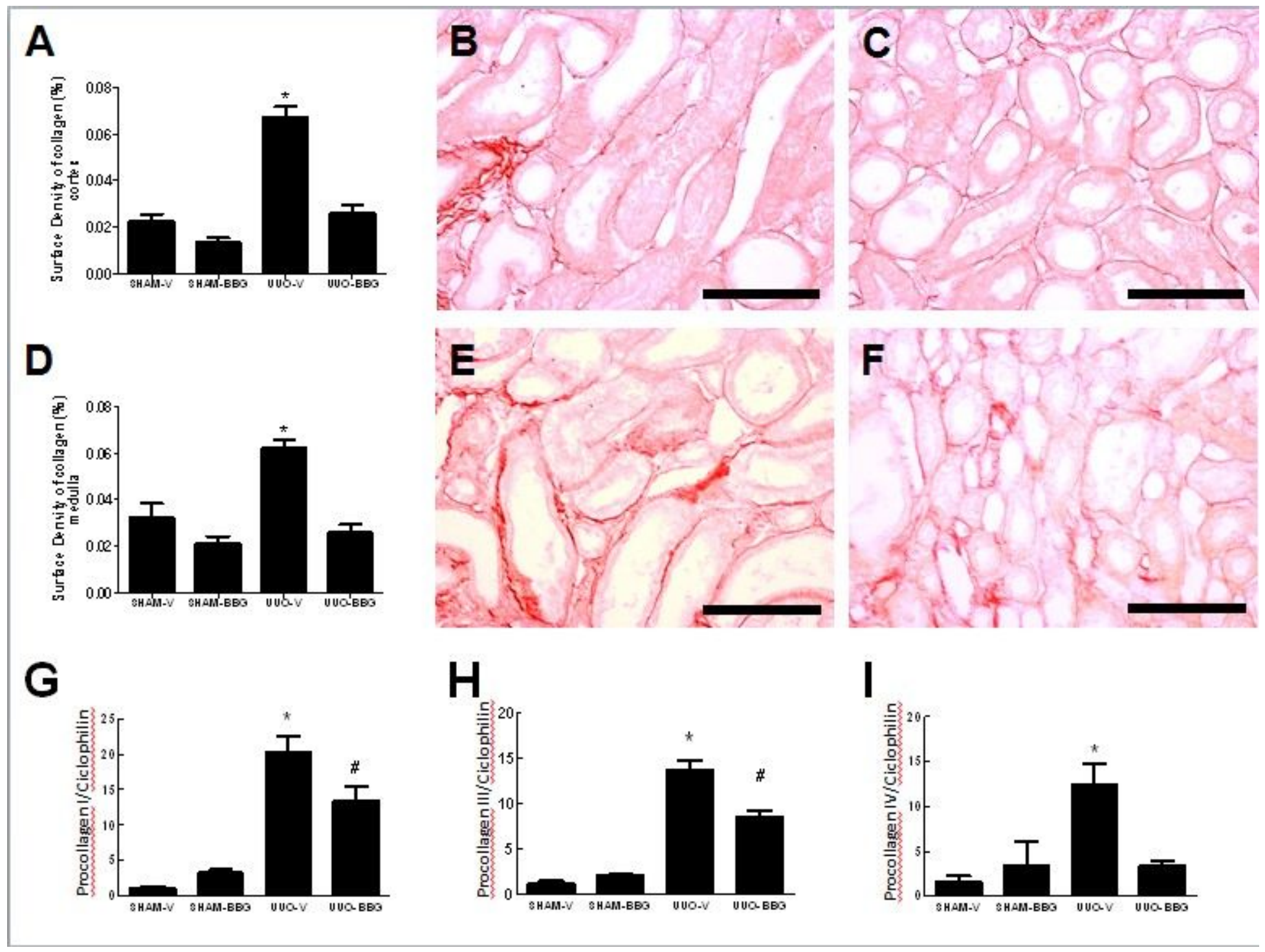

Figure 4

BBG reduces the renal collagen expression (protein and mRNA) in UUO. a Graphical representation of Sirius Red surface density in renal cortex. Columns indicate means \pm SEM of 20 captured images of each kidney in the different experimental groups. * $p<0.05$ vs UUO-BBG and both SHAM groups. $b$ Representative staining of the renal cortex from UUO-V group and c UUO-BBG group. d Graphical representation of Sirius Red surface density in renal medulla. Columns indicate means \pm SEM of 20 captured images of each kidney in the different experimental groups. * $p<0.05$ vs UUO-BBG and both SHAM groups. e Representative staining of the renal medulla from UUO-V group and $f$ UUO-BBG group. $($ Bar $=100 \mu \mathrm{m}) . \mathrm{g}$ Graphical representation of the mRNA expression of Procollagen I by semiquantitative RT-PCR. Quantification of densitometric values obtained from the ratio Procollagen I/Ciclophilin (mean \pm SEM, $n=6$ ) under the 3 experimental conditions indicated on the abscissa. ${ }^{*} p<0.05$ vs UUO-BBG and both SHAM groups. \# $p<0.05$ vs UUO-V and both SHAM groups. h Graphical representation of the mRNA expression of Procollagen III by semiquantitative RT-PCR. Quantification of densitometric values obtained from the ratio Procollagen III/Ciclophilin (mean $\pm S E M, n=6$ ) under the 3 experimental conditions indicated on the abscissa. ${ }^{*} p<0.05$ vs UUO-BBG and both SHAM groups. $\# p<0.05$ vs UUO-V and both 
SHAM groups. i Graphical representation of the mRNA expression of Procollagen IV by semiquantitative RT-PCR. Quantification of densitometric values obtained from the ratio Procollagen IV/Ciclophilin (mean \pm SEM, $n=6$ ) under the 3 experimental conditions indicated on the abscissa. * $p<0.05$ vs UUO-BBG and both SHAM groups
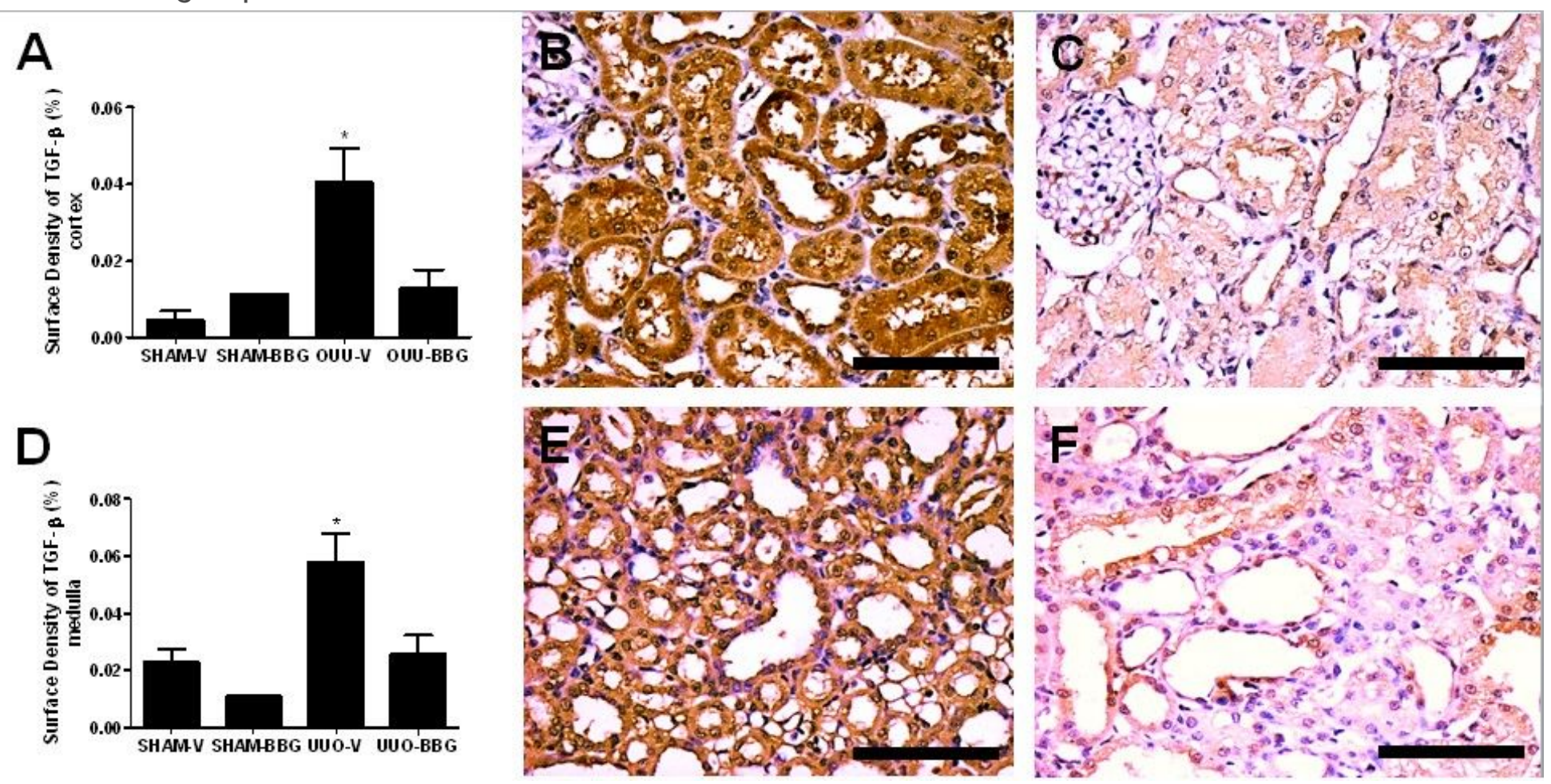

Figure 5

BBG reduces the immunoexpression of TGF- $\beta$ in UUO. a Graphical representation of TGF- $\beta$ surface density in renal cortex. Columns indicate means \pm SEM of 20 captured images of each kidney in the different experimental groups. * $p<0.05$ vs UUO-BBG and both SHAM groups. b Representative immunostaining of the renal cortex from UUO-V group and c UUO-BBG group. $d$ Graphical representation of TGF- $\beta$ surface density in renal medulla. Columns indicate means \pm SEM of 20 captured images of each kidney in the different experimental groups. ${ }^{*} p<0.05$ vs UUO-BBG and both SHAM groups. e Representative immunostaining of the renal medulla from UUO-V group and $f$ UUO-BBG group. (Bar $=100$ $\mu \mathrm{m})$ 


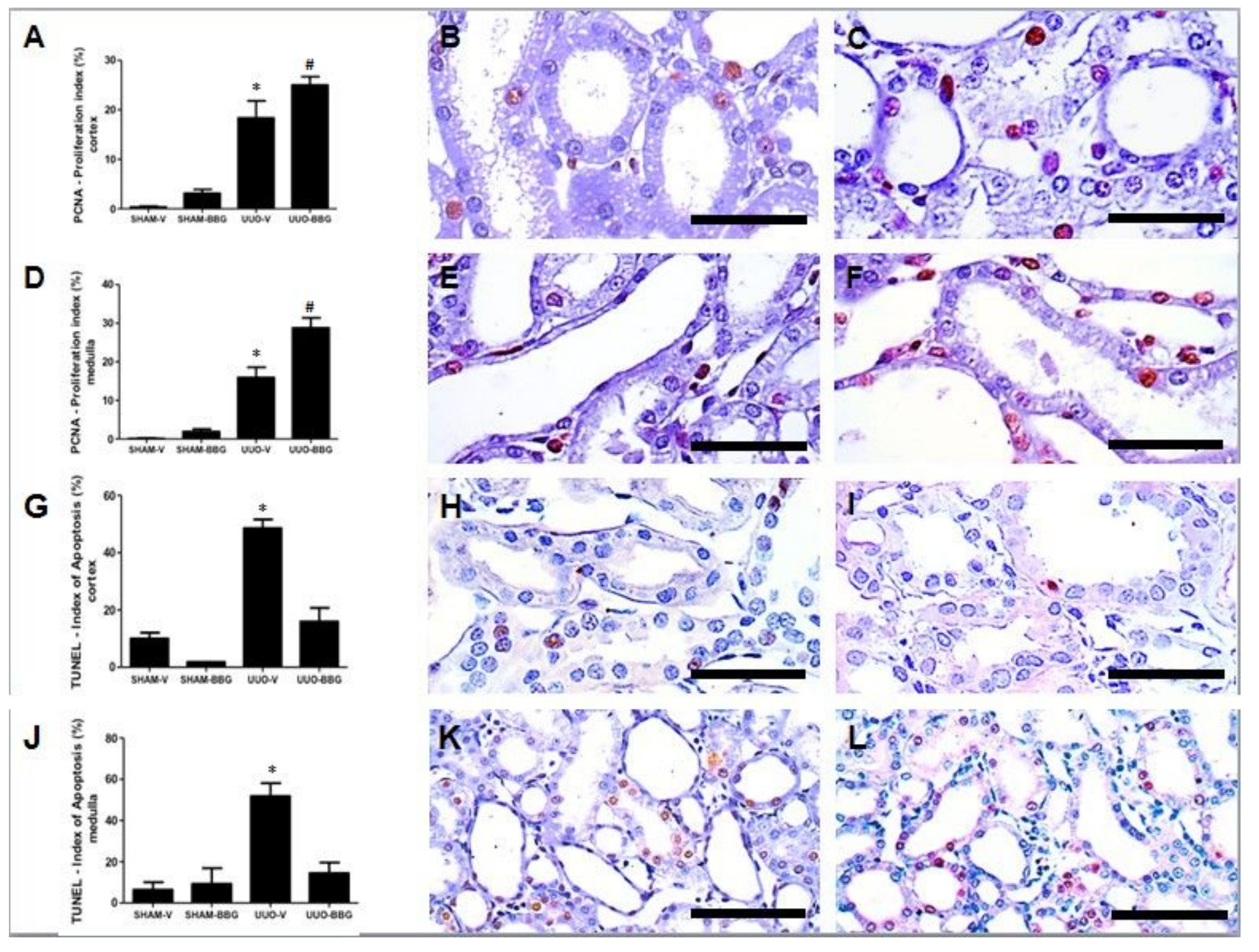

Figure 6

BBG administration induces proliferation and decreases apoptosis in tubular cells in UUO. a Graphical representation of a semi-quantitative analysis of the proliferation index (percentage of PCNA+ cells) in renal cortex. Columns indicate means \pm SEM of 20 captured images of each kidney in the different experimental groups. ${ }^{*} p<0.05$ vs UUO-BBG and both SHAM groups. $\# p<0.05$ vs UUO-V and both SHAM groups. b Representative immunostaining of the renal cortex from UUO-V and c UUO-BBG (Bar $=50 \mu m)$. d Graphical representation of a semi-quantitative analysis of the proliferation index (percentage of PCNA+ cells) in renal medulla. Columns indicate means \pm SEM of 20 captured images of each kidney in the different experimental groups. * $p<0.05$ vs UUO-BBG and both SHAM groups. $\# p<0.05$ vs UUO-V and both SHAM groups. e Representative immunostaining of the renal medulla from UUO-V and $f$ UUO-BBG $(B a r=50 \mu \mathrm{m}) . \mathrm{g}$ Graphical representations of a semi-quantitative analysis of the apoptotic index (percentage of TUNEL + cells) in renal cortex. Columns indicate means \pm SEM of 20 captured images of each kidney in the different experimental groups. * $p<0.05$ vs UUO-BBG and both SHAM groups. $h$ Representative cortical immunostaining from UUO-V and i UUO-BBG $(B a r=50 \mu \mathrm{m})$. j Graphical representations of a semi-quantitative analysis of the apoptotic index (percentage of TUNEL+ cells) in 
renal medulla. Columns indicate means \pm SEM of 20 captured images of each kidney in the different experimental groups. ${ }^{*} p<0.05$ vs UUO-BBG and both SHAM groups. $k$ Representative immunostaining in renal medulla from UUO-V and $(\mathrm{L})$ UUO-BBG $(B a r=100 \mu \mathrm{m})$

\section{Supplementary Files}

This is a list of supplementary files associated with this preprint. Click to download.

- NC3RsARRIVEGuidelinesChecklist2014.docx 